\title{
VARIANCE OF TIME TO RECRUITMENT FOR A SINGLE GRADE MANPOWER SYSTEM WITH DIFFERENT EPOCHS FOR EXITS AND TWO TYPES OF DECISIONS HAVING TWO THRESHOLDS INVOLVING TWO COMPONENTS
}

\author{
G. Ravichandran ${ }^{1}$, A. Srinivasan ${ }^{2}$ \\ ${ }^{1}$ Assistant Professor in Mathematics, TRP Engineering College (SRM GROUP), Trichy - 621 105, Tamil Nadu, India \\ ${ }^{2}$ Professor Emeritus, PG \& Research Department of Mathematics, Bishop Heber College, Trichy - 620017 ,
} Tamil Nadu, India

\begin{abstract}
In this paper, the problem of time to recruitment is studied using a univariate policy of recruitment involving optional and mandatory thresholds for a single grade manpower system where wastages (loss in manpower) occur due to attrition generated by its policy decisions and frequent breaks taken by the personnel working in the system. Assuming that (i) the policy decisions and exits occur at different epochs (ii) the number of exits form a homogeneous Poisson process (iii) both the optional and mandatory thresholds for the cumulative loss of manpower have independently a normal component due to attrition and a second component due to frequent breaks (iv) wastage due to attrition and frequent breaks form separately a sequence of independent and exponentially distributed random variables and $(v)$ inter-policy decision times are of two types, one with high rate of attrition and the other having low rate of attrition, a stochastic model is constructed and variance of time to recruitment is obtained when the inter-policy decision times form (i) a geometric process and (ii) an order statistics.
\end{abstract}

Keywords: Single Grade Manpower System; Different Decision And Exit Epochs; Two Types of Policy Decisions, Optional And Mandatory Thresholds With Two Components, Geometric Process; Order Statistics; Univariate Policy of Recruitment And Variance of Time To Recruitment.

\section{INTRODUCTION}

Wastages due to attrition are usual in any marketing organization. A judicious and planned recruitment has to be advocated as frequent recruitment is not advisable. In $[1,3]$ several stochastic models for manpower planning have been discussed. In [2] some manpower planning problems have been analyzed using statistical techniques. Assuming different epochs for decisions and exits and the number of exits form a homogeneous Poisson process, variance of time to recruitment is obtained in $[5,8,9]$ using univariate policy of recruitment and Laplace transform in the analysis according as the inter decision times are independent and identically distributed exponential random variables or forming a geometric process or an order statistics. The manpower planning problem in [5] is studied in [7] when the breakdown threshold has two components. Recently, in $[10,11,12]$ the research work in $[5,8,9]$ have been studied by considering optional and mandatory thresholds as two control limits with single(normal) component, which is a variation from the work of [4] in the context of considering non-instantaneous exits at decision epochs. In the present paper, for a single grade manpower system, a mathematical model is constructed in which attrition due to policy decisions take place at exit points and there are optional and mandatory thresholds with two components, the first component is the normal component for the cumulative wastage due to attrition and the second one is the component due to frequent breaks taken by the personnel working in the system. A univariate policy of recruitment based on shock model approach is used to determine the variance of time to recruitment when the policy decisions are classified into two types according to their intensities of attrition. The present paper extends the research work in [13] when the optional and mandatory thresholds have these two components.

\section{MODEL DESCRIPTION}

Consider a single grade organization taking decisions at random epochs in $(0, \infty)$ and at every decision making epoch a random number of persons quit the organization. There is an associated loss of manpower if a person quits. It is assumed that this wastage is linear and cumulative. Let $X_{i}$ be a continuous random variable representing the wastage caused at the $i^{\text {th }}$ exit point and $S_{k}$ be the cumulative wastage occurred in the first $\mathrm{k}$ exit points. It is assumed that $X_{i}$ 's are independent and identically distributed exponential random variables with probability density function $\mathrm{m}($.$) , distribution function \mathrm{M}($.$) and mean$ $\frac{1}{\alpha}(\alpha>0)$. Let $N_{e}(t)$ be the number of exit points lying 
in $(0, \mathrm{t}]$. Let $U_{k}$ be the continuous random variable representing the time between the $(\mathrm{k}-1)^{\text {th }}$ and $\mathrm{k}^{\text {th }}$ policy decisions. Let $W_{i}$ be the continuous random variable representing the time between the $(\mathrm{i}-1)^{\text {th }}$ and $\mathrm{i}^{\text {th }}$ exit times. It is assumed that $W_{i}$ 's are independent and identically distributed exponential random variables with probability density function $\mathrm{g}($.), distribution function $\mathrm{G}($.). Let $\mathrm{Y}$ and $\mathrm{Z}$ be the optional and mandatory threshold levels for the cumulative wastage in the organization. Let $\mathrm{Y}_{1}$ be the first component of $\mathrm{Y}$ corresponding to cumulative wastage due to attrition and $\mathrm{Y}_{2}$ be its second component corresponding to frequent breaks taken by the personnel working in the system. Let $\mathrm{Z}_{1}$ be the first component of $\mathrm{Z}$ corresponding to cumulative wastage due to attrition and $\mathrm{Z}_{2}$ be its second component corresponding to frequent breaks taken by the personnel working in the system. Then $\mathrm{Y}=\mathrm{Y}_{1}+\mathrm{Y}_{2}$ and $\mathrm{Z}=$ $\mathrm{Z}_{1}+\mathrm{Z}_{2}$. It is assumed that (i) $\mathrm{Y}<\mathrm{Z}$ (ii) $\mathrm{Y}_{1}$ and $\mathrm{Y}_{2}$ are independent and (iii) $\mathrm{Z}_{1}$ and $\mathrm{Z}_{2}$ are independent. Let $\mathrm{H}_{1}($.),
$\mathrm{H}_{2}(),. \mathrm{H}_{11}(),. \mathrm{H}_{12}(),. \mathrm{H}_{21}($.$) and \mathrm{H}_{22}($.$) be the distribution of$ $\mathrm{Y}, \mathrm{Z}, \mathrm{Y}_{1}, \mathrm{Y}_{2}, \mathrm{Z}_{1}$ and $\mathrm{Z}_{2}$ respectively. Let $\mathrm{p}$ be the probability that the organization is not going for recruitment when optional threshold is exceeded by the cumulative wastage. Let $\mathrm{q}$ be the probability that every policy decision has exit of personnel. As $q=0$ corresponds to the case where exits are impossible, it is assumed that $\mathrm{q} \neq 0$. Let $\mathrm{T}$ be the random variable denoting the time to recruitment with probability distribution $\mathrm{L}($.$) , density function l($.$) , mean \mathrm{E}(\mathrm{T})$ and variance $\mathrm{V}(\mathrm{T})$. Let $\bar{a}($.) be the Laplace transform of a(.). The univariate CUM policy of recruitment employed in this paper is stated as follows:

Recruitment is done whenever the cumulative wastage in the organization exceeds the mandatory threshold. The organization may or may not go for recruitment if the cumulative wastage exceeds the optional threshold.

\section{MAIN RESULT}

From the recruitment policy, we note that

$P(T>t)=\sum_{k=0}^{\infty} P\left[N_{e}(t)=k\right] P\left(S_{k} \leq Y\right)+p \sum_{k=0}^{\infty} P\left[N_{e}(t)=k\right] P\left(S_{k}>Y\right) P\left(S_{k} \leq Z\right)$

Assuming $\quad M(x)=1-e^{-\alpha x}, H_{11}(y)=1-e^{-\theta_{1} y}, H_{12}(y)=1-e^{-\theta_{2} y}, H_{21}(z)=1-e^{-\gamma_{1} z}, H_{22}(z)=1-e^{-\gamma_{2} z}$,

we get

$P\left(S_{k} \leq Y\right)=\left(1+N_{1}\right) b^{k}-N_{1} a^{k}$, where $a=\bar{m}\left(\theta_{1}\right), b=\bar{m}\left(\theta_{2}\right), N_{1}=\frac{\theta_{2}}{\theta_{1}-\theta_{2}}$

$P\left(S_{k}>Y\right)=1-\left(1+N_{1}\right) b^{k}+N_{1} a^{k}$

$P\left(S_{k} \leq Z\right)=\left(1+N_{2}\right) b_{1}{ }^{k}-N_{2} a_{1}{ }^{k}$, where $a_{1}=\bar{m}\left(\gamma_{1}\right), b_{1}=\bar{m}\left(\gamma_{2}\right), N_{2}=\frac{\gamma_{2}}{\gamma_{1}-\gamma_{2}}$

Substituting (3), (4) and (5) in (1), we get

$$
\begin{gathered}
P(T>t)=\sum_{k=0}^{\infty} P\left[N_{e}(t)=k\right]\left[\left(1+\mathrm{N}_{1}\right) \mathrm{b}^{\mathrm{k}}-N_{1} a^{k}\right]+p \sum_{k=0}^{\infty} P\left[N_{e}(t)=k\right]\left[1-\left(1+\mathrm{N}_{1}\right) b^{k}+N_{1} a^{k}\right]\left[\left(1+N_{2}\right) b_{1}{ }^{k}-N_{2} a_{1}{ }^{k}\right] \\
L(t)=\left(1+N_{1}\right) \bar{b} \sum_{\mathrm{k}=1}^{\infty} G_{k}(t) b^{k-1}-N_{1} \bar{a} \sum_{k=1}^{\infty} G_{k}(t) a^{k-1}+p\left\{\left(1+N_{2}\right) \overline{b_{1}} \sum_{k=1}^{\infty} G_{k}(t) b_{1}{ }^{k-1}-N_{2} \overline{a_{1}} \sum_{k=1}^{\infty} G_{k}(t) a_{1}{ }^{k-1}\right\} \\
-p\left[\left(1+N_{1}\right)\left(1+N_{2}\right) \overline{b b_{1}} \sum_{k=1}^{\infty} G_{k}(t)\left(b b_{1}\right)^{k-1}+\left(1+N_{1}\right) N_{2} \overline{a_{1} b} \sum_{k=1}^{\infty} G_{k}(t)\left(a_{1} b\right)^{k-1}\right] \\
+p\left[\left(1+N_{2}\right) N_{1} \overline{a b_{1}} \sum_{k=1}^{\infty} G_{k}(t)\left(a b_{1}\right)^{k-1}-N_{1} N_{2} \overline{a a_{1}} \sum_{k=1}^{\infty} G_{k}(t)\left(a a_{1}\right)^{k-1}\right]
\end{gathered}
$$

where $\bar{a}=1-a, \bar{b}=1-b, \overline{a_{1}}=1-a_{1}, \overline{b_{1}}=1-b_{1}, \overline{a b_{1}}=1-a b_{1}, \overline{b b_{1}}=1-b b_{1}, \overline{a_{1} b}=1-a_{1} b, \overline{a a_{1}}=1-a a_{1}$ 
From (7), we get

$$
\begin{aligned}
\bar{l}(s)=\frac{\left(1+N_{1}\right) \bar{b} \bar{g}(s)}{1-b \bar{g}(s)}-\frac{N_{1} \bar{a} \bar{g}(s)}{1-a \bar{g}(s)}+p\left\{\frac{\left(1+N_{2}\right) \overline{b_{1}} \bar{g}(s)}{1-b_{1} \bar{g}(s)}-\frac{N_{2} \overline{a_{1}} \bar{g}(s)}{1-a_{1} \bar{g}(s)}-\frac{\left(1+N_{1}\right)\left(1+N_{2}\right) \overline{b b_{1}} \bar{g}(s)}{1-b b_{1} \bar{g}(s)}+\right. \\
\left.\frac{\left(1+N_{1}\right) N_{2} \overline{a_{1} b} \bar{g}(s)}{1-a_{1} \mathrm{~b} \bar{g}(s)}+\frac{\left(1+N_{2}\right) N_{1} \overline{a b_{1}} \bar{g}(s)}{1-a b_{1} \bar{g}(s)}-\frac{N_{1} N_{2} \overline{a a_{1}} \bar{g}(s)}{1-a a_{1} \bar{g}(s)}\right\}
\end{aligned}
$$

It is known that $\mathrm{E}\left(\mathrm{T}^{r}\right)=(-1)^{r}\left[\frac{d^{r}}{d s^{r}} \bar{l}(s)\right]_{s=0}, \quad r=1,2,3, \ldots$

From (9) and (10), it can be shown that

$$
\begin{aligned}
E(T)= & -\bar{g}^{\prime}(0)\left\{\frac{1+N_{1}}{\bar{b}}-\frac{N_{1}}{\bar{a}}+p\left[\frac{1+N_{2}}{\overline{b_{1}}}-\frac{N_{2}}{\overline{a_{1}}}-\frac{\left(1+N_{1}\right)\left(1+N_{2}\right)}{\overline{b b_{1}}}+\frac{\left(1+N_{1}\right) N_{2}}{\overline{a_{1} b}}+\frac{\left(1+N_{2}\right)}{\overline{a b_{1}}} N_{1}-\frac{N_{1} N_{2}}{\overline{a a_{1}}}\right]\right\} \\
E\left(T^{2}\right)= & \left(1+N_{1}\right)\left[\frac{\bar{b}^{\prime \prime}(0)+2 b\left[\bar{g}^{\prime}(0)\right]^{2}}{(\bar{b})^{2}}\right]-N_{1}\left[\frac{\bar{a}^{\prime \prime}(0)+2 a\left[\bar{g}^{\prime}(0)\right]^{2}}{(\bar{a})^{2}}\right] \\
+ & p\left\{\frac{\left(1+N_{2}\right)\left[\overline{b_{1}} \bar{g}^{\prime \prime}(0)+2 b_{1}\left(\bar{g}^{\prime}(0)^{2}\right]\right.}{(\bar{b})^{2}}-\frac{N_{2}\left[\bar{a}_{1} \bar{g}^{\prime \prime}(0)+2 a_{1} \bar{g}^{\prime}(0)^{2}\right]}{\left(\overline{a_{1}}\right)^{2}}\right\} \\
& +p\left\{-\frac{\left(1+N_{1}\right)\left(1+N_{2}\right)\left[\overline{b b_{1}} \bar{g}^{\prime \prime}(0)+2 b b_{1}\left(\bar{g}^{\prime}(0)^{2}\right]\right.}{\left(\overline{b b_{1}}\right)^{2}}+\frac{\left(1+N_{1}\right) N_{2}\left[\overline{a_{1} b} \bar{g}^{\prime \prime}(0)+2 a_{1} b \bar{g}^{\prime}(0)^{2}\right]}{\left(\overline{a_{1} b}\right)^{2}}\right\} \\
& +p\left\{\frac{\left(1+N_{2}\right) N_{1}\left[\overline{a b_{1}} \bar{g}^{\prime \prime}(0)+2 a b_{1}\left(\bar{g}^{\prime}(0)^{2}\right]\right.}{\left(\overline{a b_{1}}\right)^{2}}-\frac{N_{1} N_{2}\left[\overline{a a_{1}} \bar{g}^{\prime \prime}(0)+2 a a_{1} a \bar{g}^{\prime}(0)^{2}\right]}{\left(\overline{a a_{1}}\right)^{2}}\right\}
\end{aligned}
$$

Variance of time to recruitment can be computed from (11) and (12).

We now determine variance of time to recruitment for two different cases on inter-policy decision times.

Case(i): $\left\{U_{k}\right\}_{k=1}^{\infty}$ form a geometric process with rate $c,(c>0)$. The distribution $F($.$) \quad of \mathrm{U}_{1} \quad$ is $F(t)=1-\left[p_{1} e^{-\lambda_{1} t}+\left(1-p_{1}\right) e^{-\lambda_{2} t}\right], \lambda_{1}, \lambda_{2}>0$, where $p_{1}$ and $\left(1-p_{1}\right)$ are proportions of policy decisions with high and low rates of attritions $\lambda_{1}$ and $\lambda_{2}$ respectively .

It can be shown that the distribution function $\mathrm{G}($.$) of the inter-exit times W satisfy the relation G(x)=q \sum_{n=1}^{\infty}(1-q)^{n-1} F_{n}(\mathrm{x})$.

Therefore $\bar{g}(s)=q \sum_{n=1}^{\infty}(1-q)^{n-1} \overline{f_{n}}(s)$, where $\overline{f_{n}}(s)=\prod_{k=1}^{n} \bar{f}\left(\frac{s}{c^{k-1}}\right)$

From (11), we get

$E(T)=-\bar{g}^{\prime}(0)\left\{\frac{1+N_{1}}{\bar{b}}-\frac{N_{1}}{\bar{a}}+p\left[\frac{1+N_{2}}{\overline{b_{1}}}-\frac{N_{2}}{\overline{a_{1}}}-\frac{\left(1+N_{1}\right)\left(1+N_{2}\right)}{\overline{b b}}+\frac{\left(1+N_{1}\right) N_{2}}{\overline{a_{1} b}}+\frac{\left(1+N_{2}\right) N_{1}}{\overline{a b}}-\frac{N_{1} N_{2}}{\overline{a a_{1}}}\right]\right\}$

From (14), we get

$\bar{g}^{\prime}(0)=\frac{c}{(c-1+q)} \bar{f}^{\prime}(0)$, where $\bar{f}^{\prime}(0)=-\left(\frac{p_{1}}{\lambda_{1}}+\frac{1-p_{1}}{\lambda_{2}}\right)$ 
From (12), we get

$$
\begin{aligned}
E\left(T^{2}\right) & =\left(1+N_{1}\right)\left[\frac{\bar{b} \bar{g}^{\prime \prime}(\mathrm{O})+2 b\left[\bar{g}^{\prime}(\mathrm{O})\right]^{2}}{(\bar{b})^{2}}\right]-N_{1}\left[\frac{\bar{a} \bar{g}^{\prime \prime}(\mathrm{O})+2 a\left[\bar{g}^{\prime}(\mathrm{O})\right]^{2}}{(\bar{a})^{2}}\right] \\
+ & p\left\{\frac{\left(1+N_{2}\right)\left[\overline{b_{1}} \bar{g}^{\prime \prime}(0)+2 b_{1}\left(\bar{g}^{\prime}(0)^{2}\right]\right.}{(\bar{b})^{2}}-\frac{N_{2}\left[\bar{a}_{1} \bar{g}^{\prime \prime}(0)+2 a_{1} \bar{g}^{\prime}(0)^{2}\right]}{\left(\overline{a_{1}}\right)^{2}}\right\} \\
& +p\left\{-\frac{\left(1+N_{1}\right)\left(1+N_{2}\right)\left[\overline{b b_{1}} \bar{g}^{\prime \prime}(0)+2 b b_{1}\left(\bar{g}^{\prime}(0)^{2}\right]\right.}{\left(\overline{b b_{1}}\right)^{2}}+\frac{\left(1+N_{1}\right) N_{2}\left[\bar{a}_{1} b \bar{g}^{\prime \prime}(0)+2 a_{1} b \bar{g}^{\prime}(0)^{2}\right]}{\left(\overline{a_{1} b}\right)^{2}}\right\} \\
& +p\left\{\frac{\left(1+N_{2}\right) N_{1}\left[\overline{a b} \bar{g}^{\prime \prime}(0)+2 a b_{1}\left(\bar{g}^{\prime}(0)^{2}\right]\right.}{\left(\overline{a b_{1}}\right)^{2}}-\frac{N_{1} N_{2}\left[\overline{a a_{1}} \bar{g}^{\prime \prime}(0)+2 a a_{1} a \bar{g}(0)^{2}\right]}{\left(\overline{a a_{1}}\right)^{2}}\right\}
\end{aligned}
$$

From (14) and on simplification, we get

$$
\bar{g}^{\prime \prime}(0)=\frac{c^{2}}{\left(c^{2}-1+q\right)} \bar{f}^{\prime \prime}(0)+\frac{2 c^{2}(1-q)}{\left(c^{2}-1+q\right)(c-1+q)}\left(\bar{f}^{\prime}(0)\right)^{2}, \text { where } \bar{f}^{\prime \prime}(0)=2\left(\frac{p_{1}}{\lambda_{1}{ }^{2}}+\frac{1-p_{1}}{\lambda_{2}^{2}}\right)
$$

and $\bar{a}, \bar{b}, \overline{a_{1}}, \overline{b_{1}}, \overline{a b_{1}}, \overline{b b_{1}}, \overline{a a_{1}}, \overline{a_{1} b}$ are given by (8).

Equations (15), (16) together with (17) and (18) give the mean and variance on the time to recruitment for case (i).

Case (ii): $\left\{U_{k}\right\}_{k=1}^{\infty}$ form an order statistics where the sample of size $\mathrm{r}$ associated with this order statistics is selected from a hyperexponential population of independent and identically distributed inter-policy decision times, where the common distribution $\mathrm{F}($.) is given as in case(i).

Let $F_{u(j)}($.$) and f_{u(j)}($.$) be the distribution and the probability density function of the j^{\text {th }}$ order statistic selected from the sample of size $\mathrm{r}$ from the exponential population $\left\{U_{k}\right\}_{k=1}^{\infty}$. From the theory of order statistics [12], it is known that

$f_{u(j)}(t)=j\left(\begin{array}{l}r \\ j\end{array}\right)[F(t)]^{j-1} f(t)[1-F(t)]^{r-j}, j=1,2, . ., r$

Suppose $f(t)=f_{u(1)}(t)$

From (10), (11) and (19), we get

$E(T)=-\frac{{\overline{f_{u(1)}}}^{\prime}(0)}{q}\left\{\frac{1+\mathrm{N}_{1}}{\bar{b}}-\frac{N_{1}}{\bar{a}}+p\left[\frac{1+N_{2}}{\bar{b}_{1}}-\frac{N_{2}}{\overline{a_{1}}}-\frac{\left(1+N_{1}\right)\left(1+N_{2}\right)}{\overline{b b_{1}}}+\frac{\left(1+N_{1}\right) N_{2}}{\overline{a_{1} b}}+\frac{\left(1+N_{2}\right) N_{1}}{\overline{a b_{1}}}-\frac{N_{1} N_{2}}{\overline{a a_{1}}}\right]\right\}$

${\overline{f_{u(1)}}}^{\prime}(0)=-A_{r}$ where $A_{r}=\sum_{n=0}^{r} \frac{{ }^{r} C_{n} p_{1}{ }^{n}\left(1-p_{1}\right)^{r-n}}{\left[\lambda_{1} n+(r-n) \lambda_{2}\right]}$

$$
\begin{aligned}
E\left(T^{2}\right)= & \frac{2\left(1+N_{1}\right)\left[\bar{b} q B_{r}-(\bar{b} q-1) A_{r}^{2}\right]}{(\bar{b} q)^{2}}-\frac{2 N_{1}\left[\bar{a} q B_{r}-(\bar{a} q-1) A_{r}^{2}\right]}{(\bar{a} q)^{2}}+ \\
& \frac{2 p\left(1+N_{2}\right)\left[\overline{b_{1}} q B_{r}-\left(\overline{b_{1}} q-1\right) A_{r}^{2}\right]}{\left(\overline{b_{1}} q\right)^{2}}-\frac{2 p N_{2}\left[\overline{a_{1}} q B_{r}-\left(\overline{a_{1}} q-1\right) A_{r}{ }^{2}\right]}{\left(\overline{a_{1}} q\right)^{2}}- \\
& \frac{2 p\left(1+N_{1}\right)\left(1+N_{2}\right)\left[\overline{b b_{1}} q B_{r}-\left(\overline{b b_{1}} q-1\right) A_{r}{ }^{2}\right]}{\left(\overline{b b_{1}} q\right)^{2}}+\frac{2 p\left(1+N_{1}\right) N_{2}\left[\overline{a_{1} b} q B_{r}-\left(\overline{a_{1} b} q-1\right) A_{r}{ }^{2}\right]}{\left(\overline{a_{1} b} q\right)^{2}}+
\end{aligned}
$$




$$
\frac{2 p\left(1+N_{2}\right) N_{1}\left[\overline{a b_{1}} q B_{r}-\left(\overline{a b_{1}} q-1\right) A_{r}^{2}\right]}{\left(\overline{a b_{1}} q\right)^{2}}-\frac{2 p N_{1} N_{2}\left[\overline{a a_{1}} q B_{r}-\left(\overline{a a_{1}} q-1\right) A_{r}^{2}\right]}{\left(\overline{a a_{1}} q\right)^{2}}
$$

$\overline{f_{u(1)}}(0)=2 B_{r}$, where $B_{r}=\sum_{n=0}^{r} \frac{{ }^{r} C_{n} p_{1}{ }^{n}\left(1-p_{1}\right)^{r-n}}{\left[\lambda_{1} n+(r-n) \lambda_{2}\right]}$

and

$\bar{a}, \bar{b}, \overline{a_{1}}, \overline{b_{1}}, \overline{a b_{1}}, \overline{b b_{1}}, \overline{a a_{1}}, \overline{a_{1} b}$ are given by $(8)$

Equations (20), (21) together with (22) and (23) give the mean and variance on the time to recruitment when $f(t)=f_{u(1)}(t)$.

Suppose $f(t)=f_{u(r)}(t)$.

From (10), (11) and (19), we get

$E(T)=-\frac{{\overline{f_{u(r)}}}^{\prime}(0)}{q}\left\{\frac{1+\mathrm{N}_{1}}{\bar{b}}-\frac{N_{1}}{\bar{a}}+p\left[\frac{1+N_{2}}{\bar{b}_{1}}-\frac{N_{2}}{\overline{a_{1}}}-\frac{\left(1+N_{1}\right)\left(1+N_{2}\right)}{\overline{b b_{1}}}+\frac{\left(1+N_{1}\right) N_{2}}{\overline{a_{1} b}}+\frac{\left(1+N_{2}\right) N_{1}}{\overline{a b_{1}}}-\frac{N_{1} N_{2}}{\overline{a a_{1}}}\right]\right\}$

${\overline{f_{u(r)}}}^{\prime}(0)=C_{r}$, where $C_{r}=\sum_{n=0}^{r} \sum_{n_{1}=0}^{r-n} \frac{{ }^{r} C_{n}(-1)^{r-n(r-n)} C_{n_{1}} p_{1}^{n_{1}}\left(1-p_{1}\right)^{r-n-n_{1}}}{\left[\lambda_{1} n_{1}+\left(r-n-n_{1}\right) \lambda_{2}\right]}$

and

$$
\begin{aligned}
E\left(T^{2}\right)= & \frac{-2\left(1+N_{1}\right)\left[\bar{b} q D_{r}+(\bar{b} q-1) C_{r}{ }^{2}\right]}{(\bar{b} q)^{2}}+\frac{2 N_{1}\left[\bar{a} q D_{r}+(\bar{a} q-1) C_{r}{ }^{2}\right]}{(\bar{a} q)^{2}}- \\
& \frac{2 p\left(1+N_{2}\right)\left[\overline{b_{1}} q D_{r}+\left(\overline{b_{1}} q-1\right) C_{r}{ }^{2}\right]}{\left(\overline{b_{1}} q\right)^{2}}+\frac{2 p N_{2}\left[\overline{a_{1}} q D_{r}+\left(\overline{a_{1}} q-1\right) C_{r}{ }^{2}\right]}{\left(\overline{a_{1}} q\right)^{2}}+ \\
& \frac{2 p\left(1+N_{1}\right)\left(1+N_{2}\right)\left[\overline{b b_{1}} q D_{r}+\left(\overline{b b_{1}} q-1\right) C_{r}{ }^{2}\right]}{\left(\overline{b b_{1}} q\right)^{2}}-\frac{2 p\left(1+N_{1}\right) N_{2}\left[\overline{a_{1} b} q D_{r}+\left(\overline{a_{1} b} q-1\right) C_{r}{ }^{2}\right]}{\left(\overline{a_{1} b} q\right)^{2}}- \\
& \frac{2 p\left(1+N_{2}\right) N_{1}\left[\overline{a b_{1}} q D_{r}+\left(\overline{a b_{1}} q-1\right) C_{r}{ }^{2}\right]}{\left(\overline{a b_{1}} q\right)^{2}}+\frac{2 p N_{1} N_{2}\left[\overline{a a_{1}} q D_{r}+\left(\overline{a a_{1}} q-1\right) C_{r}{ }^{2}\right]}{\left(\overline{a a_{1}} q\right)^{2}}
\end{aligned}
$$

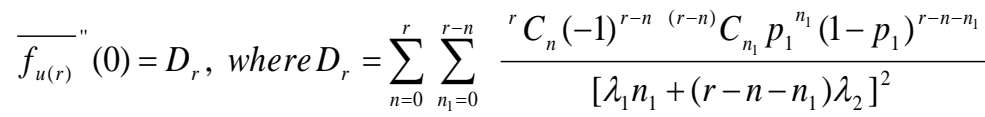

and $\bar{a}, \bar{b}, \overline{a_{1}}, \overline{b_{1}}, \overline{a b_{1}}, \overline{b b_{1}}, \overline{a a_{1}}, \overline{a_{1} b}$ are given by $(8)$.

Equations (24), (25) together with (26) and (27) give the mean and variance on the time to recruitment when $f(t)=f_{u(r)}(t)$.

\section{Remark}

Variance of time to recruitment for the case when $\left\{U_{k}\right\}_{k=1}^{\infty}$ is a sequence of independent and identically distributed hyper exponential random variables can be obtained by taking $c=1$ in case (i).

\section{FINDINGS}

From the above results, the following observations are presented which agree with reality:

1. When $\alpha$ increases and keeping all the other parameters fixed, the average wastage increases. Therefore the mean and variance of time to recruitment increase. 
2. As $\lambda$ increases, on the average, the inter-decision time decreases and consequently the mean and variance of time to recruitment decrease when the other parameters are fixed.

3. The mean and variance of the time to recruitment decrease or increase according as $c>1$ or $c<1$, since the geometric process of inter-policy decision times is stochastically decreasing when $c>1$ and increasing when $c<1$.

\section{CONCLUSION}

The models discussed in this paper are new in the context of considering (i) separate points (exit points) on the time axis for attrition, thereby removing a severe limitation on instantaneous attrition at decision epochs (ii) associating a probability for any decision to have exit points (iii) considering two types of policy decisions, one with high rate of attrition and the other having low rate of attrition and (iv) provision of optional and mandatory thresholds with two components, a normal component due to attrition and a second component due to frequent breaks. From the organization's point of view, our models are more suitable than the corresponding models with instantaneous attrition at decision epochs, as the provision of exit points at which attrition actually takes place, postpone the time to recruitment.

\section{REFERENCES}

[1] Bartholomew, D. J., Stochastic model for social processes, New York, John Wiley and Sons, 1973.

[2] Bartholomew, D. J., and Andrew Forbes, F., Statistical techniques for manpower planning, New York, John Wiley and Sons, 1979.

[3] Grinold, R. C., and Marshall, K.T, Manpower planning models, New York, North-Holland, 1977.

[4] Esther Clara, J. B., Contributions to the study on some stochastic models in manpower planning, doctoral diss., Bharathidasan University, Tiruchirappalli, 2012.

[5] Devi, A., and Srinivasan, A., Variance of time to recruitment for single grade manpower system with different epochs for decisions and exits, International Journal of Research in Mathematics and Computations, vol. 2, issue 1, 2014, pp. 23 - 27.

[6] Medhi, J., Stochastic processes New Delhi, Wiley Eastern, second edition, 1994.

[7] Devi, A., and Srinivasan, A., Determination of expected time to recruitment for a single grade manpower system with different epochs for decisions and exits when the breakdown threshold has two components, International Journal of technical Research and Applications, vol. 3, issue 5, , 2015, pp. $210-214$.

[8] Devi, A., and Srinivasan, A., A stochastic model for time to recruitment in a single grade manpower system with different epochs for decisions and exits having inter- decision times as geometric process, Second International Conference on Business Analytic and Intelligence, ( ICBAI ), 2014.
[9] Devi, A., and Srinivasan, A., Expected time to recruitment for a single grade manpower system with different epochs for decisions and exits with interdecision times as an order statistics, International Journal of Innovative Research in Science, Engineering and Technology, vol. 4, issue 8, , 2015, pp. 7517-7520.

[10] Ravichandran, G., and Srinivasan, A., Variance of time to recruitment for a single grade manpower system with two thresholds having different epochs for decisions and exits, Indian Journal of Applied Research, vol. 5, issue 1, 2015, pp. $60-64$.

[11] Ravichandran, G., and Srinivasan, A., Time to recruitment for a single grade manpower system with two thresholds, different epochs for exits and geometric inter-decisions, IOSR Journal of Mathematics, vol. 11, issue 2, Ver. III, 2015, pp. $29-32$.

[12] Ravichandran, G., and Srinivasan, A., Time to recruitment for a single grade manpower system with two thresholds, different epochs for inter-decisions as an order statistics and exits, International Journal Of Engineering And Science, vol. 5, issue 6, 2015, pp. 24 -28 .

[13] Ravichandran, G., and Srinivasan, A., Variance of time to recruitment for a single grade manpower system with different epochs for exits and two types of decisions having two thresholds, International Journal of Innovative Research in Science, Engineering and Technology, vol. 5, issue 3, 2016, pp. $3801-3806$. 